EUROPEAN JOURNAL OF PURE AND APPLIED MATHEMATICS

Vol. 13, No. 1, 2020, 113-129

ISSN 1307-5543 - www.ejpam.com

Published by New York Business Global

\title{
Anti fuzzy interior ideals on Ordered AG-groupoids
}

\author{
Nasreen Kausar ${ }^{1, *}$, Meshari Alesemi², Salahuddin ${ }^{2}$ \\ 1 Department of Mathematics, University of Agriculture FSD Pakistan \\ 2 Department of Mathematics, Jazan University, Jazan, Kingdom of Saudi Arabia
}

\begin{abstract}
The purpose of this paper is to investigate, the characterizations of different classes of non-associative ordered semigroups by using anti fuzzy left (resp. right, interior) ideals.
\end{abstract}

2020 Mathematics Subject Classifications: 13Cxx, 94D05, 13Axx, $18 \mathrm{~B} 40$

Key Words and Phrases: Fuzzy sets, anti fuzzy AG-subgroupoids, anti fuzzy left (resp. right, interior) ideals, left (resp. right, weakly, intra-, (2,2)-) regular ordered AG-groupoids.

\section{Introduction}

In 1972, a generalization of commutative semigroup has been established by Naseeruddin et al. [14]. In ternary commutative law, $a b c=c b a$, they introduced the braces on the left side of this law and explored a new pseudo associative law, that is $(a b) c=(c b) a$. This they called the left invertive law. A groupoid $S$ is a left almost semigroup (abbreviated as LA-semigroup), if it satisfies the left invertive law: $(a b) c=(c b) a$. This structure is also known as Abel-Grassmann's groupoid (abbreviated as AG-groupoid) by Protic et al. [27]. In fact an AG-groupoid is non-commutative and non-associative semigroup. Ideals in AG-groupoids have been investigated in [26].

In [6] (resp. [3]), a groupoid $S$ is said to be medial (resp. paramedial) if $(a b)(c d)=$ $(a c)(b d)$ (resp. $(a b)(c d)=(d b)(c a))$. In [14], an AG-groupoid is medial, but in general an AG-groupoid needs not to be paramedial. However by Protic et al. [27], every AGgroupoid with left identity is paramedial and also satisfies $a(b c)=b(a c),(a b)(c d)=$ $(d c)(b a)$.

In [15], if $(S, \cdot, \leq)$ is an ordered semigroup and $A \subseteq S$, we define $(A]=\{s \in S: s \leq a$ for some $a \in A\}$. A non-empty subset $A$ of $S$ is an ordered subsemigroup of $S$ if $A^{2} \subseteq A$.

The notions of ideals play a crucial role in the study of (ring, semiring, near-ring, semigroup, ordered semigroup) theory etc.

* Corresponding author.

DOI: https://doi.org/10.29020/nybg.ejpam.v13i1.3576

Email addresses: kausar.nasreen57@gmail.com (K. Nasreen)

malesemi@jazanu.edu.sa (M. Alesemi), drsalah12@hotmail.com (Salahuddin) 
A non-empty subset $A$ of $S$ is a left (resp. right) ideal of $S$, if following hold (1) $S A \subseteq A$ (resp. $A S \subseteq A$ ). (2) If $a \in A$ and $b \in S$ such that $b \leq a$ implies $b \in A$. Equivalent definition: $A$ is a left ( resp. right) ideal of $S$ if $(A] \subseteq A$ and $S A \subseteq A$ (resp. $A S \subseteq A$ ).

A non-empty subset $A$ of $S$ is an interior ideal of $S$ if (1) $S A S \subseteq A$. (2) If $a \in A$ and $b \in S$ such that $b \leq a$ implies $b \in A$.

In $[17,18]$, an ordered semigroup $S$ is said to be regular, if for every $a \in S$, there exists $x \in S$ such that $a \leq a x a$. Equivalent definitions are as follows: (1) $A \subseteq(A S A]$ for every $A \subseteq S$. (2) $a \in(a S a]$ for every $a \in S$. An ordered semigroup $S$ is said to be (2,2)-regular, if for every $a \in S$, there exists $x \in S$ such that $a \leq a^{2} x a^{2}$. Equivalent definitions are as follows: (1) $A \subseteq\left(A^{2} S A^{2}\right]$ for every $A \subseteq S$. (2) $a \in\left(a^{2} S a^{2}\right]$ for every $a \in S$.

An ordered semigroup $S$ is said to be weakly regular, if for every $a \in S$, there exist $x, y \in S$ such that $a \leq$ axay. Equivalent definitions are as follows: $(1) A \subseteq\left((A S)^{2}\right]$ for every $A \subseteq S$. (2) $a \in\left((a S)^{2}\right]$ for every $a \in S$.

In $[16,18]$, an ordered semigroup $S$ is an intra-regular if for every $a \in S$ there exist $x, y \in S$ such that $a \leq x a^{2} y$. Equivalent definitions are as follows: (1) $A \subseteq\left(S A^{2} S\right]$ for every $A \subseteq S$. (2) $a \in\left(S a^{2} S\right]$ for every $a \in S$.

We define anti fuzzy left (resp. right, interior) ideals in an ordered AG-groupoids, basically an ordered AG-groupoid is non-commutative and non-associative ordered semigroup.

In this present paper, we characterize regular (resp. right regular, left regular, $(2,2)$ regular, weakly regular and intra-regular) ordered AG-groupoids in terms of anti fuzzy left (resp. right, interior) ideals. In this regard, we prove that in (regular, right regular, weakly regular ) ordered AG-groupoids, the concept of anti fuzzy ( interior, two-sided) ideals coincide. The concept of anti fuzzy (interior, two-sided) ideals coincide in $((2,2)$, left, intra-) regular ordered AG-groupoids with left identity.

\section{Preliminaries}

In [31], an ordered AG-groupoid $S$, is a partially ordered set, at the same time an AGgroupoid such that $a \leq b$, implies $a c \leq b c$ and $c a \leq c b$ for all $a, b, c \in S$. Two conditions are equivalent to the one condition $(c a) d \leq(c b) d$, for all $a, b, c, d \in S$. An ordered AG-groupoid is also called a po-AG-groupoid for short.

Example 1. Consider a set $S=\{e, f, a, b, c\}$ with the following multiplication "” and order relation " $\leq$ ":

$$
\begin{aligned}
& \text { - } \begin{array}{lllll}
e & f & a & b & c
\end{array} \\
& \begin{array}{llllll}
e & e & f & a & b & c
\end{array} \\
& \begin{array}{llllll}
f & f & f & f & b & c
\end{array} \\
& \begin{array}{llllll}
a & a & f & c & b & c
\end{array} \\
& \begin{array}{lllllll}
b & c & c & c & f & b
\end{array} \\
& \begin{array}{llllll}
c & b & b & b & c & f
\end{array} \\
& \leq:=\{(e, e),(e, a),(e, b),(e, c),(f, f),(f, b),(f, c),(a, a),(a, c),(b, b),(b, c),(c, c)\} .
\end{aligned}
$$

Then $(S, \cdot, \leq)$ is an ordered $A G$-groupoid with left identity $e$. 
Let $S$ be an ordered AG-groupoid and $A \subseteq S$, we define a subset $(A]=\{s \in S: s \leq a$ for some $a \in A\}$ of $S$ and obviously $A \subseteq(A]$. If $A=\{a\}$, then we write (a] instead of $(\{a\}]$. For $A, B \subseteq S$, then $A B=\{a b \mid a \in A, b \in B\}, \quad((A]]=(A],(A](B] \subseteq(A B]$, $((A]) B]]=(A B]$, if $A \subseteq B$ then $(A] \subseteq(B],(A \cap B] \neq(A] \cap(B]$ in general.

For $\emptyset \neq A \subseteq S$. $A$ is an ordered AG-subgroupoid of $S$ if $A^{2} \subseteq A$. $A$ is left (resp. right) ideal of $S$ if (1) $S A \subseteq A$ (resp. $A S \subseteq A$ ). (2) if $a \in A$ and $b \in S$ such that $b \leq a$ implies $b \in A$.

Equivalent definition: $A$ is left (resp. right) ideal of $S$ if $(A] \subseteq A$ and $S A \subseteq A$ (resp. $A S \subseteq A$ ). $A$ is an ideal of $S$ if $A$ is both a left and a right ideal of $S$. If $A, B$ are ideals of $S$, then $A \cup B$ and $A \cap B$ are also ideals of $S$.

A non-empty subset $A$ of an ordered AG-groupoid $S$ is an interior ideal of $S$ if (1) $(S A) S \subseteq A$. (2) If $a \in A$ and $b \in S$ such that $b \leq a$ implies $b \in A$ (or $(A] \subseteq A$ ).

An ordered AG-groupoid $S$ is left ( resp. right) regular, if for every $a \in S$, there exists $x \in S$ such that $a \leq x a^{2}$ (resp. $a \leq a^{2} x$ ). Equivalent definitions are as follows: (1) $A \subseteq\left(S A^{2}\right]$ (resp. $\left.A \subseteq\left(A^{2} S\right]\right)$ for every $A \subseteq S$. (2) $a \in\left(S a^{2}\right]$ (resp. $a \in\left(a^{2} S\right]$ ) for every $a \in S$.

An ordered AG-groupoid $S$ is regular, if for every $a \in S$, there exists $x \in S$ such that $a \leq(a x) a$. Equivalent definitions: (1) $A \subseteq((A S) A]$ for every $A \subseteq S$. (2) $a \in((a S) a]$ for every $a \in S$.

An ordered AG-groupoid $S$ is completely regular, if it is regular, left regular, right regular.

An ordered AG-groupoid $S$ is strongly regular, if for every $a \in S$, there exists $x \in S$ such that $a \leq(a x) a$ and $a x=x a$.

Every strongly regular ordered AG-groupoid is right regular ordered AG-groupoid.

An ordered AG-groupoid $S$ is said to be weakly regular, if for every $a \in S$, there exist $x, y \in S$ such that $a \leq(a x)(a y)$. Equivalent definitions are as follows: $(1) A \subseteq\left((A S)^{2}\right]$ for every $A \subseteq S$. (2) $a \in\left((a S)^{2}\right]$ for every $a \in S$.

An ordered AG-groupoid $S$ is an intra-regular, if for every $a \in S$, there exist $x, y \in S$ such that $a \leq\left(x a^{2}\right) y$. Equivalent definitions are as follows: (1) $A \subseteq\left(\left(S A^{2}\right) S\right]$ for every $A \subseteq S .(2) a \in\left(\left(S a^{2}\right) S\right]$ for every $a \in S$.

We denote by $L(a), R(a), I(a)$ the left ideal, the right ideal and the ideal of $S$, respectively generated by $a$. We have $L(a)=\{s \in S: s \leq a$ or $s \leq x a$ for some $x \in S\}=(a \cup S a], R(a)=(a \cup a S], I(a)=(a \cup S a \cup a S \cup(S a) S]$.

Example 2. Let $S=\{a, b, c, d, e\}$. Define multiplication "” in $S$ as follows :

$\begin{array}{llllll}\cdot & a & b & c & d & e \\ a & a & a & a & a & a \\ b & a & a & a & a & a \\ c & a & a & e & c & d \\ d & a & a & d & e & c \\ e & a & a & c & d & e\end{array}$

and $\leq:=\{(a, a),(b, b),(c, c),(d, d),(e, e)\}$. Then $S$ is an ordered AG-groupoid. $A=$ $\{c, d, e\}$ is an $A G$-subgroupoid of $S$ and $I=\{a, c, d, e\}$ is an ideal of $S$. 
Remark 1. Every ideal (whether right, left or two-sided) is an AG-subgroupoid but the converse is not true in general.

An ordered AG-groupoid $S$ is to be locally associative, if $(a . a) . a=a .(a . a)$ for every $a \in S$.

Example 3. Let $S=\{a, b, c\}$. Define multiplication "” in $S$ as follows :

$\begin{array}{llll}\cdot & a & b & c \\ a & c & c & b \\ b & b & b & b \\ c & b & b & b\end{array}$

and $\leq:=\{(a, a),(b, b),(c, c)\}$. Then $(S, \cdot, \leq)$ is a locally associative ordered AG-groupoid.

In a locally associative ordered AG-groupoids $S$, we define powers of an element as follow: $a^{1}=a, a^{n+1}=a^{n} a$. If $S$ has a left identity $e$, we define $a^{0}=e$, as left identity is unique in an ordered AG-groupoid.A locally associative ordered AG-groupoid $S$ with left identity $e$ has associative powers.

\section{Anti fuzzy interior ideals on ordered AG-groupoids}

A fuzzy set $\mu$ on a given set $X$ is described as an arbitrary function $\mu: X \rightarrow[0,1]$, where $[0,1]$ is the unit closed interval of real numbers.

The fundamental concept of a fuzzy set, introduced by Zadeh in his classic paper [33] 1965, which gives a natural frame work for the generalizations of some basic notions of algebra, for example set (resp. semigroup, group, ring, near-ring, semiring) theory, groupoids, real analysis, topology, differential equations and so forth. Rosenfeld [29], introduced the concept of fuzzy set in groups. The study of fuzzy set in semigroups investigated by Kuroki [21-23]. He studied fuzzy (interior, bi-, quasi-, semiprime quasi) ideals in semigroups. Dib and Galham in [4], examined the definition of fuzzy groupoid (resp. semigroup). They studied fuzzy ideals and fuzzy bi-ideals of fuzzy semigroups. A systematic exposition of fuzzy semigroups by Mordeson et al. appeared in [24], where one can find theoretical results on fuzzy semigroups and their use in fuzzy finite state machines and fuzzy languages. Fuzzy sets in ordered semigroups/ordered groupoids established by Kehayopulu and Tsingelis [19]. They also studied fuzzy bi-ideals and fuzzy quasi-ideals in ordered semigroups $[19,20]$.

In [2], Biswas introduced the concept of anti fuzzy subgroups of groups and studied the basic properties of groups in terms of anti fuzzy subgroups. Hong and Jun [5] modified the Biswas idea and applied it into BCK-algebra. Akram and Dar defined anti fuzzy left h-ideals of hemiring and discussed the basic properties of hemiring [1].

By a fuzzy set $\mu$ of an ordered AG-groupoid $S$, we mean a function $\mu: S \rightarrow[0,1]$ and the complement of $\mu$ is denoted by $\mu^{\prime}$, is a fuzzy set in $S$ given by $\mu^{\prime}(x)=1-\mu(x)$ for all $x \in S$. 
A fuzzy set $\mu$ of an ordered AG-groupoid $S$ is an anti fuzzy AG-subgroupoid of $S$ if $\mu(x y) \leq \max \{\mu(x), \mu(y)\}$ for all $x, y \in S$.

$\mu$ is an anti fuzzy left (resp. right) ideal of $S$, if (1) $\mu(x y) \leq \mu(y)$ (resp. $\mu(x y) \leq$ $\mu(x))$. (2) $x \leq y$, implies $\mu(x) \leq \mu(y)$ for all $x, y \in S . \mu$ is an anti fuzzy ideal of $S$, if $\mu$ is both an anti fuzzy left ideal and an anti fuzzy right ideal of $S$. Equivalently, $\mu$ is an anti fuzzy ideal of $S$ if $(1) \mu(x y) \leq \max \{\mu(x), \mu(y)\}$. (2) $x \leq y$, implies $\mu(x) \leq \mu(y)$ for all $x, y \in S$.

Every anti fuzzy ideal (whether left, right, two-sided) is an anti fuzzy AG-subgroupoid but the converse is not true in general.

A fuzzy set $\mu$ of $S$ is an anti fuzzy interior ideal of $S$, if (1) $\mu((x a) y) \leq \mu(a)$. (2) $x \leq y$, implies $\mu(x) \leq \mu(y)$ for all $x, a, y \in S$.

We denote by $F(S)$, the set of all fuzzy subsets of $S$. We define an order relation " $\subseteq$ " on $F(S)$ such that $f \subseteq g$ if and only if $f(x) \leq g(x)$ for all $x \in S$. Then $(F(S), \circ, \subseteq)$ is an ordered AG-groupoid.

For $f \wedge g$ and $f \vee g$, we define $(f \wedge g)(x)=\min \{f(x), g(x)\}$ and $(f \vee g)(x)=$ $\max \{f(x), g(x)\}$.

For $a \in S$, we define $A_{a}=\{(y, z) \in S \times S \mid a \leq y z\}$. Let $f$ and $g$ be fuzzy subsets of $S$, the product $f \circ g$ of $f$ and $g$ is defined by:

$$
(f \circ g)(a)= \begin{cases}\wedge_{(y, z) \in A_{a}} \max \{f(y), g(z)\} & \text { if } A_{a} \neq \emptyset \\ 0 & \text { if } A_{a}=\emptyset\end{cases}
$$

For a non-empty family of fuzzy subsets $\left\{f_{i}\right\}_{i \in I}$, of $S$, the fuzzy subsets $\vee_{i \in I} f_{i}$ and $\wedge_{i \in I} f_{i}$ of $S$ are defined as follows:

$$
\begin{aligned}
\left(\vee_{i \in I} f_{i}\right)(a) & :=\sup _{i \in I}\left\{f_{i}(a)\right\} \\
\text { and }\left(\wedge_{i \in I} f_{i}\right)(a) & : \quad=\inf _{i \in I}\left\{f_{i}(a)\right\} .
\end{aligned}
$$

If $I$ is a finite set, say $I=\{1,2, \ldots n\}$, then clearly,

$$
\begin{aligned}
\vee_{i \in I} f_{i}(a) & =\max \left\{f_{1}(a), f_{2}(a), \ldots, f_{n}(a)\right\} \\
\text { and } \wedge_{i \in I} f_{i}(a) & =\min \left\{f_{1}(a), f_{2}(a), \ldots, f_{n}(a)\right\}
\end{aligned}
$$

For $S$, the fuzzy subsets " 0 " and "1" are defined as $0(x):=0$ and $1(x):=1$.

$$
\begin{aligned}
& 0: \quad S \rightarrow[0,1], x \mapsto 0(x):=0 . \\
& 1: \quad S \rightarrow[0,1], x \mapsto 1(x):=1 .
\end{aligned}
$$

Clearly, the fuzzy subset " 0 "(resp. "1") of $S$ is the least ( resp. the greatest) element of the ordered set $(F(S), \leq)$. The fuzzy subset "0" is the zero element of $(F(S), \circ, \leq)$ (that is, $f \circ 0=0 \circ f=0$ and $0 \leq f$ for every $f \in F(S)$ ).

For $\emptyset \neq A \subseteq S$, the anti characteristic function of $A$ is denoted by $\chi_{A}^{C}$ and defined as

$$
\chi_{A}^{C}(a)=\left\{\begin{array}{l}
0 \text { if } a \in A \\
1 \text { if } a \notin A
\end{array}\right.
$$


An ordered AG-groupoid $S$ can be considered a fuzzy subset of itself and we write $S=\chi_{S}^{C}$, i.e., $S(x)=\chi_{S}^{C}(x)=0$ for all $x \in S$. This implies that $S(x)=0$ for all $x \in S$. $\chi_{(A B]}^{C}$

For $A, B \subseteq S$, then $A \subseteq B$ if and only if $\chi_{A}^{C} \geq \chi_{B}^{C}, \chi_{A}^{C} \cap \chi_{B}^{C}=\chi_{A \cap B}^{C}$ and $\chi_{A}^{C} \circ \chi_{B}^{C}=$

Let $\mu$ be a fuzzy subset of $S$, then for all $t \in(0,1]$, we define a set $L(\mu ; t)=\{x \in S \mid$ $\mu(x) \leq t\}$, which is called lower $t$-level set of $\mu$ and can be used for the characterization of $\mu$.

Example 4. Let $S=\{a, b, c, d\}$. Define multiplication "” in $S$ as follows :

$\begin{array}{lllll}\cdot & a & b & c & d \\ a & c & d & a & b \\ b & b & c & d & a \\ c & a & b & c & d \\ d & d & a & b & c\end{array}$

and $\leq:=\{(a, a),(b, b),(c, c),(d, d)\}$. Then $S$ is an ordered AG-groupoid. Let $\mu$ be a fuzzy subset of $S$. We define $\mu(a)=\mu(c)=0.7, \mu(b)=\mu(d)=0$. Hence $\mu$ is an anti fuzzy $A G$-subgroupoid of $S$.

Example 5. Let $S=\{a, b, c, d\}$. Define multiplication "” in $S$ as follows :

$\begin{array}{lllll}\cdot & a & b & c & d \\ a & a & a & a & a \\ b & a & a & a & a \\ c & a & a & d & a \\ d & a & a & c & d\end{array}$

and $\leq:=\{(a, a),(b, b),(c, c),(d, d)\}$. Then $S$ is an ordered AG-groupoid. Let $\mu$ be a fuzzy subset of $S$. We define $\mu(a)=\mu(c)=\mu(d)=0, \mu(b)=0.7$. Hence $\mu$ is an anti fuzzy right ideal of $S$.

Remark 2. Example 4 and Example 5 show that, every anti fuzzy ideal (whether right, left, two-sided) is an anti fuzzy AG-subgroupoid, but the converse is not true.

Lemma 1. Let $S$ be an ordered $A G$-groupoid and $\emptyset \neq A \subseteq S$. Then the anti characteristic function $\chi_{(A]}^{C}$ of $(A]$ is a fuzzy subset of $S$ satisfying the condition $x \leq y \Rightarrow \chi_{(A]}^{C}(x) \leq$ $\chi_{(A]}^{C}(y)$ for all $x, y \in S$.

Proof. By the definition, $\chi_{(A]}^{C}$ is a mapping of $S$ into $\{0,1\} \subseteq[0,1]$. Let $x \leq y, x, y \in S$. If $y \notin(A]$, by definition $\chi_{(A]}^{C}(y)=1$, thus $\chi_{(A]}^{C}(x) \leq \chi_{(A]}^{C}(y)$. If $y \in(A]$, by definition $\chi_{(A]}^{C}(y)=0$. Since $y \in(A]$, so there exists $z \in A$ such that $y \leq z$. Thus $x \leq z$, i.e., $x \in(A]$ and $\chi_{(A]}^{C}(x)=0$. Hence $\chi_{(A]}^{C}(x) \leq \chi_{(A]}^{C}(y)$. 
Proposition 1. Let $S$ be an ordered $A G$-groupoid and $\emptyset \neq A \subseteq S$. Then $A=(A]$ if and only if fuzzy subset $\chi_{A}^{C}$ of $S$ has the property $x \leq y \Rightarrow \chi_{A}^{C}(x) \leq \chi_{A}^{C}(y)$ for all $x, y \in S$.

Proof. Suppose $A=(A]$, then the anti characteristic function $\chi_{A}^{C}$ of $A$ is a fuzzy subset of $S$ satisfying the condition $x \leq y \Rightarrow \chi_{A}^{C}(x) \leq \chi_{A}^{C}(y)$, by the Lemma 1 .

Conversely, let $x \in(A]$, this imply that there exists $y \in A$ such that $x \leq y$. By the given condition, we have $\chi_{A}^{C}(x) \leq \chi_{A}^{C}(y)$. Since $y \in A$, we have $\chi_{A}^{C}(y)=0$. Thus $\chi_{A}^{C}(x)=0$, i.e., $x \in A$. Hence $A=(A]$.

Lemma 2. Let $S$ be an ordered $A G$-groupoid and $\emptyset \neq A \subseteq S$. Then $A$ is an $A G$ subgroupoid of $S$ if and only if the anti characteristic function $\chi_{A}^{C}$ of $A$ is an anti fuzzy $A G$-subgroupoid of $S$.

Proof. Suppose $A$ is an AG-subgroupoid of $S$ and $x, y \in S$. If $x, y \notin A$, by definition $\chi_{A}^{C}(x)=1=\chi_{A}^{C}(y)$. Thus $\chi_{A}^{C}(x y) \leq \chi_{A}^{C}(x) \vee \chi_{A}^{C}(y)$. If $x, y \in A$, by definition $\chi_{A}^{C}(x)=$ $0=\chi_{A}^{C}(y) . x y \in A, A$ being an AG-subgroupoid of $S$, this imply that $\chi_{A}^{C}(x y)=0$. Thus $\chi_{A}^{C}(x y) \leq \chi_{A}^{C}(x) \vee \chi_{A}^{C}(y)$. Hence the anti characteristic function $\chi_{A}^{C}$ of $A$ is an anti fuzzy AG-subgroupoid of $S$.

Conversely, let $x y \in A^{2}, x, y \in A$. By definition of anti characteristic function $\chi_{A}^{C}(x)=$ $0=\chi_{A}^{C}(y) . \chi_{A}^{C}(x y) \leq \chi_{A}^{C}(x) \vee \chi_{A}^{C}(y)=0, \chi_{A}^{C}$ being an anti fuzzy AG-subgroupoid of $S$. This imply that $\chi_{A}^{C}(x y)=0$, i.e., $x y \in A$. Hence $A$ is an AG-subgroupoid of $S$.

Lemma 3. Let $S$ be an ordered $A G$-groupoid and $\emptyset \neq A \subseteq S$. Then $A$ is a left (resp. right) ideal of $S$ if and only if the anti characteristic function $\chi_{A}^{C}$ of $A$ is an anti fuzzy left (resp. right) ideal of $S$.

Proof. Suppose $A$ is a left ideal of $S$ and $x, y \in S$ such that $x \leq y$. This imply that $A=(A], A$ being a left ideal of $S$. Then $\chi_{A}^{C}(x) \leq \chi_{A}^{C}(y)$, by the Proposition 1. If $y \notin A$, by definition $\chi_{A}^{C}(y)=1$. Thus $\chi_{A}^{C}(x y) \leq \chi_{A}^{C}(y)$. If $y \in A$, by definition $\chi_{A}^{C}(y)=0$. $x y \in A, A$ being a left ideal, so $\chi_{A}^{C}(x y)=0$. Thus $\chi_{A}^{C}(x y) \leq \chi_{A}^{C}(y)$. Hence the anti characteristic function $\chi_{A}^{C}$ of $A$ is an anti fuzzy left ideal of $S$.

Conversely, let $y \in A$ and $x \in S$ such that $x \leq y$. This imply that $\chi_{A}^{C}(x) \leq \chi_{A}^{C}(y)$, $\chi_{A}^{C}$ being an anti fuzzy left ideal of $S$. Then $A=(A]$, by the Proposition 1. Let $x y \in S A$, where $y \in A, x \in S$. By definition of anti characteristic function $\chi_{A}^{C}(y)=0 . \chi_{A}^{C}(x y) \leq$ $\chi_{A}^{C}(y)=0, \chi_{A}^{C}$ being an anti fuzzy left ideal of $S$. Thus $\chi_{A}^{C}(x y)=0$, i.e., $x y \in A$. Hence $A$ is a left ideal of $S$.

Proposition 2. Let $S$ be an ordered $A G$-groupoid and $\emptyset \neq A \subseteq S$. Then $A$ is an interior ideal of $S$ if and only if the anti characteristic function $\chi_{A}^{C}$ of $A$ is an anti fuzzy interior ideal of $S$.

Proof. Suppose $A$ is an interior ideal of $S$ and $a, x, y \in S$ such that $x \leq y$. This imply that $A=(A], A$ being an interior-ideal. Then $\chi_{A}^{C}(x) \leq \chi_{A}^{C}(y)$, by the Proposition 1. If $a \notin A$, by definition $\chi_{A}^{C}(a)=1$. Thus $\chi_{A}^{C}((x a) y) \leq \chi_{A}^{C}(a)$. If $a \in A$, by definition 
$\chi_{A}^{C}(a)=0 .(x a) y \in A, A$ being an interior ideal, this imply that $\chi_{A}^{C}((x a) y)=0$. Thus $\chi_{A}^{C}((x a) y) \leq \chi_{A}^{C}(a)$. Hence the anti characteristic function $\chi_{A}^{C}$ of $A$ is an anti fuzzy interior ideal of $S$.

Conversely, let $y \in A$ and $x \in S$ such that $x \leq y$. This imply that $\chi_{A}^{C}(x) \leq \chi_{A}^{C}(y)$, $\chi_{A}^{C}$ being an anti fuzzy interior ideal of $S$. Then $A=(A]$, by the Proposition 1 . Let $t \in(S A) S$, implies $t=(x a) y$, where $a \in A$ and $x, y \in S$. By definition of anti characteristic function $\chi_{A}^{C}(a)=0 . \quad \chi_{A}^{C}((x a) y) \leq \chi_{A}^{C}(a)=0, \chi_{A}^{C}$ being an anti fuzzy interior ideal of $S$. Thus $\chi_{A}^{C}((x a) y)=0$, i.e., $(x a) y \in A$. Hence $A$ is an interior ideal of $S$.

Lemma 4. Let $\mu$ be a fuzzy subset of an ordered AG-groupoid $S$. Then $\mu$ is an anti fuzzy $A G$-subgroupoid of $S$ if and only if lower t-level $L(\mu ; t)$ of $\mu$ is an $A G$-subgroupoid of $S$ for all $t \in(0,1]$.

Proof. Suppose $\mu$ is an anti fuzzy AG-subgroupoid of $S$ and $x, y \in L(\mu ; t)$, this imply that $\mu(x), \mu(y) \leq t . \mu(x y) \leq \mu(x) \vee \mu(y) \leq t, \mu$ being an anti fuzzy AG-subgroupoid, i.e., $x y \in L(\mu ; t)$. Hence $L(\mu ; t)$ is an AG-subgroupoid of $S$.

Conversely, we have to show that $\mu(x y) \leq \mu(x) \vee \mu(y), x, y \in S$. We suppose a contradiction $\mu(x y)>\mu(x) \wedge \mu(y)$. Assume $\mu(x)=t=\mu(y)$, this imply that $\mu(x), \mu(y) \leq t$, i.e., $x, y \in L(\mu ; t)$. But $\mu(x y)>t$, i.e., $x y \notin U(\mu ; t)$, which is a contradiction. Hence $\mu(x y) \leq \mu(x) \vee \mu(y)$.

Lemma 5. Let $\mu$ be a fuzzy subset of an ordered AG-groupoid $S$. Then $\mu$ is an anti fuzzy left (resp. right) ideal of $S$ if and only if lower $t$-level $L(\mu ; t)$ of $\mu$ is a left (resp. right) ideal of $S$ for all $t \in(0,1]$.

Proof. Suppose $\mu$ is an anti fuzzy left ideal of $S$. Let $y \in L(\mu ; t)$ and $x \in S$ such that $x \leq y$, this imply that $\mu(y) \leq t . \mu(x) \leq \mu(y) \leq t$ and $\mu(x y) \leq \mu(y) \leq t, \mu$ being an anti fuzzy left ideal of $S$. Thus $x, x y \in L(\mu ; t)$. Hence $L(\mu ; t)$ is a left ideal of $S$.

Conversely, suppose $L(\mu ; t)$ is a left ideal of $S$ and $x, y \in S$ such that $x \leq y$. We have to show that $\mu(x) \leq \mu(y)$ and $\mu(x y) \leq \mu(y)$. We suppose a contradiction $\mu(x)>\mu(y)$ and $\mu(x y)>\mu(y)$. Let $\mu(y)=t$, this imply that $\mu(y) \leq t$, i.e., $y \in L(\mu ; t)$. But $\mu(x)>t$ and $\mu(x y)>t$, i.e., $x, x y \notin L(\mu ; t)$, which is a contradiction. Hence $\mu(x) \leq \mu(y)$ and $\mu(x y) \leq \mu(y)$.

Proposition 3. Let $\mu$ be a fuzzy subset of an ordered AG-groupoid $S$. Then $\mu$ is an anti fuzzy interior ideal of $S$ if and only if the lower t-level $L(\mu ; t)$ of $\mu$ is an interior ideal of $S$ for all $t \in(0,1]$.

Proof. Suppose $\mu$ is an anti fuzzy interior ideal of $S$. Let $y \in L(\mu ; t)$ and $x \in S$ such that $x \leq y$, this imply that $\mu(y) \leq t . \mu(x) \leq \mu(y) \leq t, \mu$ being an anti fuzzy interior ideal of $S$. Thus $\mu(x) \leq t$, i.e., $x \in L(\mu ; t)$. Let $a \in L(\mu ; t)$ and $x, y \in S$, by definition $\mu(a) \leq t . \mu((x a) y) \leq \mu(a) \leq t, \mu$ being an anti fuzzy interior ideal of $S$. Thus $\mu((x a) y) \leq t$, i.e., $(x a) y \in L(\mu ; t)$. Hence $L(\mu ; t)$ is an interior ideal of $S$. 
Conversely, suppose $L(\mu ; t)$ is an interior ideal of $S$ and $x, y, a \in S$ such that $x \leq y$. We have to show that $\mu(x) \leq \mu(y)$, we suppose a contradiction $\mu(x)>\mu(y)$. Let $\mu(y)=t$, this imply that $\mu(y) \leq t$, i.e., $y \in L(\mu ; t)$. But $\mu(x)>t$, i.e., $x \notin L(\mu ; t)$, which is a contradiction. Hence $\mu(x) \leq \mu(y)$. We have to show that $\mu((x a) y) \leq \mu(a)$, we suppose a contradiction $\mu((x a) y)>\mu(a)$. Let $\mu(a)=t$, this imply that $\mu(a) \leq t$, i.e., $a \in L(\mu ; t)$. But $\mu((x a) y)>t$, i.e., $(x a) y \notin L(\mu ; t)$, which is a contradiction. Hence $\mu((x a) y) \leq \mu(a)$.

Lemma 6. Every anti fuzzy right ideal of an ordered AG-groupoid $S$ with left identity e, is an anti fuzzy ideal of $S$.

Proof. Let $\mu$ be an anti fuzzy right ideal of $S$ and $x, y \in S$. Now $\mu(x y)=\mu((e x) y)=$ $\mu((y x) e) \leq \mu(y x) \leq \mu(y)$. Hence $\mu$ is an anti fuzzy ideal of $S$.

Remark 3. The concept of anti fuzzy (right, two-sided) ideals coincide in ordered AG-groupoids $S$ with left identity.

Lemma 7. Every anti fuzzy ideal of an ordered AG-groupoid $S$ is an anti fuzzy interior ideal of $S$.

Proof. Let $\mu$ be an anti fuzzy two-sided ideal of $S$ and $x, a, y \in S$. Now $\mu((x a) y) \leq$ $\mu(x a) \leq \mu(a)$. Hence $\mu$ is an anti fuzzy interior ideal of $S$.

Proposition 4. Let $S$ be an ordered AG-groupoid with left identity e. Then $\mu$ is an anti fuzzy interior ideal if and only if $\mu$ is an anti fuzzy ideal of $S$.

Proof. Let $\mu$ be an anti fuzzy interior ideal of $S$ and $x, y \in S$. Now $\mu(x y)=\mu((e x) y) \leq$ $\mu(x)$. Thus $\mu$ is an anti fuzzy right ideal of $S$. Hence $\mu$ is an anti fuzzy ideal of $S$ by Lemma 6. Converse is true by Lemma 7 .

Lemma 8. Every anti fuzzy right ideal of a regular ordered AG-groupoid S, is an anti fuzzy ideal of $S$.

Proof. Let $\mu$ be an anti fuzzy right ideal of $S$ and $x, y \in S$, this imply that there exists $a \in S$ such that $x \leq(x a) x$. Now $\mu(x y) \leq \mu(((x a) x) y)=\mu((y x)(x a)) \leq \mu(y x) \leq \mu(y)$. Hence $\mu$ is an anti fuzzy ideal of $S$.

Remark 4. The concept of anti fuzzy (right, two-sided) ideals coincide in regular ordered AG-groupoids $S$.

Proposition 5. Let $S$ be a regular ordered AG-groupoid. Then $\mu$ is an anti fuzzy interior ideal if and only if $\mu$ is an anti fuzzy ideal of $S$.

Proof. Let $\mu$ be an anti fuzzy interior ideal of $S$ and $x, y \in S$, this imply that there exists $a \in S$ such that $x \leq(x a) x$. Now $\mu(x y) \leq \mu(((x a) x) y)=\mu((y x)(x a)) \leq \mu(x)$. Thus $\mu$ is an anti fuzzy right ideal of $S$. Hence $\mu$ is an anti fuzzy ideal of $S$ by Lemma 8 . Converse is true by Lemma 7 . 
Lemma 9. Every anti fuzzy right (resp. left) ideal of (2,2)-regular ordered AG-groupoid $S$, is an anti fuzzy ideal of $S$.

Proof. Let $\mu$ be an anti fuzzy right ideal of $S$ and $x, y \in S$, this imply that there exists $a \in S$ such that $x \leq\left(x^{2} a\right) x^{2}$. Now $\mu(x y) \leq \mu\left(\left(\left(x^{2} a\right) x^{2}\right) y\right)=\mu\left(\left(y x^{2}\right)\left(x^{2} a\right)\right) \leq \mu\left(y x^{2}\right) \leq$ $\mu(y)$. Hence $\mu$ is an anti fuzzy ideal of $S$.

Let $\mu$ be an anti fuzzy left ideal of $S$. Now $\mu(x y) \leq \mu\left(\left(\left(x^{2} a\right) x^{2}\right) y\right)=\mu\left(\left(y x^{2}\right)\left(x^{2} a\right) \leq\right.$ $\mu((x x) a)=\mu((a x) x) \leq \mu(x)$. Hence $\mu$ is an anti fuzzy ideal of $S$.

Remark 5. The concept of anti fuzzy (right, left, two-sided) ideals coincide in $(2,2)-$ regular ordered AG-groupoids $S$.

Proposition 6. Let $S$ be a $(2,2)$-regular ordered AG-groupoid with left identity e. Then $\mu$ is an anti fuzzy interior ideal if and only if $\mu$ is an anti fuzzy ideal of $S$.

Proof. Let $\mu$ be an anti fuzzy interior ideal of $S$ and $x, y \in S$, this imply that there exists $a \in S$ such that $x \leq\left(x^{2} a\right) x^{2}$. Now

$$
\begin{aligned}
\mu(x y) & \leq \mu\left(\left(\left(x^{2} a\right) x^{2}\right) y\right)=\mu\left(\left(y x^{2}\right)\left(x^{2} a\right)\right) \leq \mu\left(x^{2}\right) \\
& =\mu(x x)=\mu((e x) x) \leq \mu(x) .
\end{aligned}
$$

Thus $\mu$ is an anti fuzzy right ideal of $S$. Hence $\mu$ is an anti fuzzy ideal of $S$ by Lemma 9 . Converse is true by Lemma 7 .

Lemma 10. Let $S$ be a right regular ordered AG-groupoid. Then every anti fuzzy right (resp. left) ideal of $S$ is an anti fuzzy ideal of $S$.

Proof. Let $\mu$ be an anti fuzzy right ideal of $S$ and $x, y \in S$, this imply that there exists $a \in S$ such that $x \leq x^{2} a$. Now

$$
\begin{aligned}
\mu(x y) & \leq \mu\left(\left(x^{2} a\right) y\right)=\mu(((x x) a) y)=\mu(((a x) x) y) \\
& =\mu((y x)(a x)) \leq \mu(y x) \leq \mu(y) .
\end{aligned}
$$

Hence $\mu$ is an anti fuzzy ideal of $S$.

Let $\mu$ be an anti fuzzy left ideal of $S$. Now

$$
\begin{aligned}
\mu(x y) & \leq \mu\left(\left(x^{2} a\right) y\right)=\mu(((x x) a) y)=\mu(((a x) x) y) \\
& =\mu((y x)(a x)) \leq \mu(a x) \leq \mu(x) .
\end{aligned}
$$

Hence $\mu$ is an anti fuzzy ideal of $S$.

Remark 6. The concept of anti fuzzy (right, left, two-sided) ideals coincide in right regular ordered $A G$-groupoids $S$.

Proposition 7. Let $S$ be a right regular ordered AG-groupoid. Then $\mu$ is an anti fuzzy interior ideal if and only if $\mu$ is an anti fuzzy ideal of $S$. 
Proof. Let $\mu$ be an anti fuzzy interior ideal of $S$ and $x, y \in S$, this imply that there exists $a \in S$ such that $x \leq x^{2} a$. Now $\mu(x y) \leq \mu\left(\left(x^{2} a\right) y\right)=\mu(((x x) a) y)=\mu(((a x) x) y) \leq \mu(x)$. Thus $\mu$ is an anti fuzzy right ideal of $S$. Hence $\mu$ is an anti fuzzy ideal of $S$ by Lemma 10 . Converse is true by Lemma 7 .

Lemma 11. Let $S$ be a left regular ordered AG-groupoid with left identity $e$. Then every anti fuzzy right (resp. left) ideal of $S$ is an anti fuzzy ideal of $S$.

Proof. Let $\mu$ be an anti fuzzy right ideal of $S$ and $x, y \in S$, this imply that there exists $a \in S$ such that $x \leq a x^{2}$. Now

$$
\begin{aligned}
\mu(x y) & \leq \mu\left(\left(a x^{2}\right) y\right)=\mu((a(x x)) y)=\mu((x(a x)) y) \\
& =\mu((y(a x)) x) \leq \mu(y(a x)) \leq \mu(y) .
\end{aligned}
$$

Hence $\mu$ is an anti fuzzy ideal of $S$.

Let $\mu$ be an anti fuzzy left ideal of $S$. Now

$$
\begin{aligned}
\mu(x y) & \leq \mu\left(\left(a x^{2}\right) y\right)=\mu((a(x x)) y)=\mu((x(a x)) y) \\
& =\mu((y(a x)) x) \leq \mu((a x) x) \leq \mu(x) .
\end{aligned}
$$

Hence $\mu$ is an anti fuzzy ideal of $S$.

Remark 7. The concept of anti fuzzy (right, left, two-sided) ideals coincide in left regular ordered AG-groupoids $S$ with left identity.

Proposition 8. Let $S$ be a left regular ordered AG-groupoid with left identity e. Then $\mu$ is an anti fuzzy interior ideal if and only if $\mu$ is an anti fuzzy ideal of $S$.

Proof. Let $\mu$ be an anti fuzzy interior ideal of $S$ and $x, y \in S$, this imply that there exists $a \in S$ such that $x \leq a x^{2}$. Now

$$
\begin{aligned}
\mu(x y) & \leq \mu\left(\left(a x^{2}\right) y\right)=\mu((a(x x)) y) \\
& =\mu((x(a x)) y)=\mu(((e x)(a x)) y) \\
& =\mu(((x x)(a e)) y)=\mu((((a e) x) x) y) \leq \mu(x) .
\end{aligned}
$$

Thus $\mu$ is an anti fuzzy right ideal of $S$. Hence $\mu$ is an anti fuzzy ideal of $S$ by Lemma 11. Converse is true by Lemma 7.

Theorem 1. Let $S$ be a right regular locally associative ordered AG-groupoid with left identity $e$. Then for every anti fuzzy interior ideal $\mu$ of $S, \mu\left(a^{n}\right)=\mu\left(a^{2 n}\right)$, where $n$ is any positive integer, for all $a \in S$.

Proof. For $n=1$. Let $a \in S$, this imply that there exists $x \in S$ such that $a \leq a^{2} x$. Thus $\mu(a) \leq \mu\left(a^{2} x\right)=\mu\left(\left(e a^{2}\right) x\right) \leq \mu\left(a^{2}\right) \leq \max \{\mu(a), \mu(a)\}=\mu(a), \quad(\mu$ is an anti fuzzy ideal of $S$ by Proposition 7). Hence $\mu(a)=\mu\left(a^{2}\right)$. Now $a^{2}=a a \leq\left(a^{2} x\right)\left(a^{2} x\right)=a^{4} x^{2}$, then 
the result is true for $n=2$. Suppose that result is true for $n=k$, i.e., $\mu\left(a^{k}\right)=\mu\left(a^{2 k}\right)$. Now $a^{k+1}=a^{k} a \leq\left(a^{2 k} x^{k}\right)\left(a^{2} x\right)=a^{2(k+1)} x^{(k+1)}$. Thus

$$
\begin{aligned}
\mu\left(a^{k+1}\right) & \leq \mu\left(a^{2(k+1)} x^{(k+1)}\right)=\mu\left(\left(e a^{2(k+1)}\right) x^{(k+1)}\right) \\
& \leq \mu\left(a^{2(k+1)}\right)=\mu\left(a^{2 k+2}\right)=\mu\left(a^{k+1} a^{k+1}\right) \\
& \leq \max \left\{\mu\left(a^{k+1}\right), \mu\left(a^{k+1}\right)\right\}=\mu\left(a^{k+1}\right) .
\end{aligned}
$$

Therefore $\mu\left(a^{k+1}\right)=\mu\left(a^{2(k+1)}\right)$. Hence by induction method, the result is true for all positive integers.

Lemma 12. Let $S$ be a right regular locally associative ordered AG-groupoid with left identity $e$. Then for every anti fuzzy interior ideal $\mu$ of $S, \mu(a b)=\mu(b a)$ for all $a, b \in S$.

Proof. Let $a, b \in S$. By using Theorem (for $n=1$ ). Now

$$
\begin{aligned}
\mu(a b) & =\mu\left((a b)^{2}\right)=\mu((a b)(a b)) \\
& =\mu((b a)(b a))=\mu\left((b a)^{2}\right)=\mu(b a) .
\end{aligned}
$$

Theorem 2. Let $S$ be a regular and right regular locally associative ordered AG-groupoid with left identity $e$. Then for every anti fuzzy interior ideal $\mu$ of $S, \mu\left(a^{n}\right)=\mu\left(a^{3 n}\right)$, where $n$ is any positive integer, for all $a \in S$.

Proof. For $n=1$. Let $a \in S$, this imply that there exists $x \in S$ such that $a \leq(a x) a$ and $a \leq a^{2} x$. Now $a \leq(a x) a \leq(a x)\left(a^{2} x\right)=a^{3} x^{2}$. Thus

$$
\begin{aligned}
\mu(a) & \leq \mu\left(a^{3} x^{2}\right)=\mu\left(\left(e a^{3}\right) x^{2}\right) \leq \mu\left(a^{3}\right) \\
& =\mu\left(a a^{2}\right) \leq \max \left\{\mu(a), \mu\left(a^{2}\right)\right\} \\
& \leq \max \{\mu(a), \mu(a), \mu(a)\}=\mu(a) .
\end{aligned}
$$

Hence $\mu(a)=\mu\left(a^{3}\right)$. Now $a^{2}=a a \leq\left(a^{3} x^{2}\right)\left(a^{3} x^{2}\right)=a^{6} x^{4}$, then the result is true for $n=2$. Suppose that result is true for $n=k$, i.e., $\mu\left(a^{k}\right)=\mu\left(a^{3 k}\right)$. Now $a^{k+1}=a^{k} a \leq$ $\left(a^{3 k} x^{2 k}\right)\left(a^{3} x^{2}\right)=a^{3(k+1)} x^{2(k+1)}$. Thus

$$
\begin{aligned}
\mu\left(a^{k+1}\right) & \leq \mu\left(a^{3(k+1)} x^{2(k+1)}\right)=\mu\left(\left(e a^{3(k+1)}\right) x^{2(k+1)}\right) \leq \mu\left(a^{3(k+1)}\right) \\
& \left.=\mu\left(a^{3 k+3}\right)=\mu\left(a^{k+1} a^{2 k+2}\right) \leq \max \mu\left(a^{k+1}\right), \mu\left(a^{2 k+2}\right)\right\} \\
& \leq \max \left\{\mu\left(a^{k+1}\right), \mu\left(a^{k+1}\right), \mu\left(a^{k+1}\right)\right\}=\mu\left(a^{k+1}\right) .
\end{aligned}
$$

Therefore $\mu\left(a^{k+1}\right)=\mu\left(a^{3(k+1)}\right)$. Hence by induction method, the result is true for all positive integers.

Lemma 13. Let $S$ be a weakly regular ordered AG-groupoid. Then every anti fuzzy right (resp. left) ideal is an anti fuzzy ideal of $S$. 
Proof. Let $\mu$ be an anti fuzzy right ideal of $S$ and $x, y \in S$, this imply that there exist $a, b \in S$ such that $x \leq(x a)(x b)$. Now

$$
\begin{aligned}
\mu(x y) & \leq \mu(((x a)(x b)) y)=\mu((((x b) a) x) y) \\
& =\mu((((a b) x) x) y)=\mu((y x)((a b) x)) \\
& =\mu((y x)(n x)) \text { say } a b=n \\
& \leq \mu(y x) \leq \mu(y) .
\end{aligned}
$$

Hence $\mu$ is an anti fuzzy ideal of $S$.

Let $\mu$ be an anti fuzzy left ideal of $S$. Now

$$
\begin{aligned}
\mu(x y) & \leq \mu(((x a)(x b)) y)=\mu((((x b) a) x) y) \\
& =\mu((((a b) x) x) y)=\mu((y x)((a b) x)) \\
& =\mu((y x)(n x)) \text { say } a b=n \\
& \leq \mu(n x) \leq \mu(x) .
\end{aligned}
$$

Hence $\mu$ is an anti fuzzy ideal of $S$.

Remark 8. The concept of anti fuzzy (right, left, two-sided) ideals coincide in weakly regular ordered $A G$-groupoids $S$.

Proposition 9. Let $S$ be a weakly regular ordered AG-groupoid. Then $\mu$ is an anti fuzzy interior ideal if and only if $\mu$ is an anti fuzzy ideal of $S$.

Proof. Let $\mu$ be an anti fuzzy interior ideal of $S$ and $x, y \in S$, this imply that there exist $a, b \in S$ such that $x \leq(x a)(x b)$. Now $\mu(x y) \leq \mu(((x a)(x b)) y)=\mu(((x b) a) x) y) \leq$ $\mu(x)$. Thus $\mu$ is an anti fuzzy right ideal of $S$. Hence $\mu$ is an anti fuzzy ideal of $S$ by Lemma 13. Converse is true by Lemma 7 .

Theorem 3. Let $S$ be an ordered AG-groupoid with left identity e. Then $S$ is a weakly regular if and only if $S$ is completely regular.

Proof. Suppose $S$ is a weakly regular ordered AG-groupoid. Let $a \in S$, then there exist $x, y \in S$ such that $a \leq(a x)(a y)$. Now $a \leq(a x)(a y)=(a a)(x y)=a^{2} t$, for some $t \in S$, this imply that $a \leq a^{2} t$. Thus $S$ is a right regular ordered AG-groupoid.

Now $a \leq(a x)(a y)=(y x)(a a)=t a^{2}$, for some $t \in S$, this imply that $a \leq t a^{2}$. Thus $S$ is a left regular ordered AG-groupoid. Now

$$
\begin{aligned}
a & \leq(a x)(a y)=(a a)(x y)=a^{2} t=(a a) t=(t a) a \\
& \leq\left(t\left(t a^{2}\right)\right) a=(t(t(a a))) a=(t(a(t a))) a \\
& =(a(t(t a))) a=(a s) a, \text { say } t(t a)=s
\end{aligned}
$$

This imply that $a \leq(a s) a$, for some $s \in S$. Thus $S$ is a regular ordered AG-groupoid. Hence $S$ is a completely regular ordered AG-groupoid. 
Conversely, let $S$ be a completely regular ordered AG-groupoid. Let $a \in S$, then there exists $x \in S$ such that $a \leq(a x) a, a \leq a^{2} x$ and $a \leq x a^{2}$. Now

$$
\begin{aligned}
a & \leq(a x) a \leq(a x)\left(x a^{2}\right)=(a x)(x(a a)) \\
& =(a x)(a(x a))=(a x)(a y), \text { say } x a=y
\end{aligned}
$$

This imply that $a \leq(a x)(a y)$, for some $x, y \in S$. Hence $S$ is weakly regular ordered AG-groupoid.

Lemma 14. Every anti fuzzy right ideal of an intra-regular ordered AG-groupoid $S$ is an anti fuzzy ideal of $S$.

Proof. Let $\mu$ be an anti fuzzy right ideal of $S$ and $x, y \in S$, this imply that there exist $a, b \in S$ such that $x \leq\left(a x^{2}\right) b$. Now $\mu(x y) \leq \mu\left(\left(\left(a x^{2}\right) b\right) y\right)=\mu\left((y b)\left(a x^{2}\right)\right) \leq \mu(y b) \leq \mu(y)$. Hence $\mu$ is an anti fuzzy ideal of $S$.

Remark 9. The concept of anti fuzzy (right, two-sided) ideals coincide in intraregular ordered AG-groupoids $S$.

Proposition 10. Let $S$ be an intra-regular ordered AG-groupoid with left identity e. Then $\mu$ is an anti fuzzy interior ideal if and only if $\mu$ is an anti fuzzy ideal of $S$.

Proof. Let $\mu$ be an anti fuzzy interior ideal of $S$ and $x, y \in S$, this imply that there exist $a, b \in S$ such that $x \leq\left(a x^{2}\right) b$. Now

$$
\begin{aligned}
x y & \leq\left(\left(a x^{2}\right) b\right) y=(y b)\left(a x^{2}\right)=n(a(x x))=n(x(a x)), \text { say } y b=n \\
& =(e n)(x(a x))=(e x)(n(a x))=(e x) m, \text { say } n(a x)=m
\end{aligned}
$$

Thus $\mu(x y) \leq \mu((e x) m) \leq \mu(x)$. Hence $\mu$ is an anti fuzzy ideal of $S$. Converse is true by Lemma 7 .

Theorem 4. Let $S$ be an intra-regular locally associative ordered AG-groupoid. Then for every anti fuzzy interior ideal $\mu$ of $S, \mu\left(a^{n}\right)=\mu\left(a^{2 n}\right)$, where $n$ is any positive integer, for all $a \in S$.

Proof. For $n=1$. Let $a \in S$, this imply that there exist $x, y \in S$ such that $a \leq\left(x a^{2}\right) y$. Thus $\mu(a) \leq \mu\left(\left(x a^{2}\right) y\right) \leq \mu\left(a^{2}\right)=\mu(a a) \leq \max \{\mu(a), \mu(a)\}=\mu(a),(\mu$ is an anti fuzzy ideal of $S$ by Proposition 10). Hence $\mu(a)=\mu\left(a^{2}\right)$. Now $a^{2}=a a \leq\left(\left(x a^{2}\right) y\right)\left(\left(x a^{2}\right) y\right)=$ $\left(\left(x a^{2}\right)\left(x a^{2}\right)\right) y^{2}=\left(x^{2} a^{4}\right) y^{2}$, then the result is true for $n=2$. Suppose that the result is true for $n=k$, i.e., $\mu\left(a^{k}\right)=\mu\left(a^{2 k}\right)$. Now $a^{k+1}=a^{k} a \leq\left(\left(x^{k} a^{2 k}\right) y^{k}\right)\left(\left(x a^{2}\right) y\right)=$ $\left(x^{k+1} a^{2(k+1)}\right) y^{k+1}$. Thus

$$
\begin{aligned}
\mu\left(a^{k+1}\right) & \leq \mu\left(\left(x^{k+1} a^{2(k+1)}\right) y^{k+1}\right) \leq \mu\left(a^{2(k+1)}\right)=\mu\left(a^{(k+1)} a^{(k+1)}\right) \\
& \leq \max \left\{\mu\left(a^{(k+1)}\right), \mu\left(a^{(k+1)}\right)\right\}=\mu\left(a^{(k+1)}\right) .
\end{aligned}
$$

Therefore $\mu\left(a^{k+1}\right)=\mu\left(a^{2(k+1)}\right)$. Hence by induction method, the result is true for all positive integers. 
Lemma 15. Let $S$ be an intra-regular locally associative ordered AG-groupoid with left identity $e$. Then for every anti fuzzy interior ideal $\mu$ of $S, \mu(a b)=\mu(b a)$ for all $a, b \in S$.

Proof. Same as Lemma 12.

\section{References}

[1] M. Akram and K. H. Dar, On anti fuzzy left h-ideals in hemirings, Int. Math. Forum, $2(2007)$ 2295-2304.

[2] R. Biswas, Fuzzy subgroups and anti fuzzy subgroups, Fuzzy sets and systems, 35(1990) 121-124.

[3] R. J. Cho, J. Jezek and T. Kepka praha, Paramedial groupoids, Czechoslovak Math. J., 49(1999) 391-399.

[4] K. A. Dib and N. Galham, Fuzzy ideals and fuzzy bi-ideals in fuzzy semigroups, Fuzzy sets and system, 92(1997) 203-215.

[5] S. M. Hong and Y. B. Jun, Anti fuzzy ideals in BCK-algebra, Kyungpook Math. J., 38(1998) 145-150.

[6] J. Jezek and T. Kepka, Medial groupoids, Rozpravy CSAV Rada mat. a prir. ved 93/2, 1983, 93 pp.

[7] T. Kadir, In discrepancy between the traditional Fuzzy logic and inductive, International Journal of Advanced and Applied Sciences, 1(2014) 36-43.

[8] N. Kausar, M. Waqar, Characterizations of non-associative rings by their intuitionistic fuzzy bi-ideals, European Journal of Pure and Applied Mathematics, Vol. 12, 1(2019) 226-250.

[9] N. Kausar, Characterizations of non-associative ordered semigroups by the properties of their fuzzy ideals with thresholds $(\alpha, \beta]$, Prikladnaya Diskretnaya Matematika, Vol. 43(2019) 37-59.

[10] N. Kausar, Direct product of finite intuitionistic fuzzy normal subrings over nonassociative rings, European Journal of Pure and Applied Mathematics, Vol. 12, 2(2019) 622-648.

[11] N. Kausar, B. Islam, M. Javaid, S, Amjad, U. Ijaz, Characterizations of nonassociative rings by the properties of their fuzzy ideals, Journal of Taibah University for Science, Vol. 13, 1(2019) 820-833.

[12] N. Kausar, B. Islam, S. Amjad, M. Waqar, Intuitionistics fuzzy ideals with thresholds $(\alpha, \beta]$ in LA-rings, European Journal of Pure and Applied Mathematics, Vol. 12, 3(2019) 906-943. 
[13] N. Kausar, M. Waqar, Direct product of finite fuzzy normal subrings over nonassociative rings, International Journal of Analysis and Applications, Vol. 17, 5(2019) $752-770$.

[14] M. A. Kazim and M. Naseeruddin, On almost semigroups, Alig. Bull. Math., 2(1972) $1-7$.

[15] N. Kehayopulu, On weakly prime ideals of ordered semigroups, Math. Japon., 35(1990) 1051-1056.

[16] N. Kehayopulu, On intra-regular ordered semigroups, Semigroup Forum, 46(1993) 271-278.

[17] N. Kehayopulu, On regular ordered semigroups, Math. Japon., 45(1997) 549-553.

[18] N. Kehayopulu, On completely regular ordered semigroups, Sci. Math., 1(1998) 27-32.

[19] N. Kehayopulu and M. Tsingelis, Fuzzy sets in ordered groupoids, Semigroup Forum, $65(2002) 128-132$.

[20] N. Kehayopulu and M. Tsingelis, Fuzzy bi-ideals in ordered semigroups, Inform Sci., 171(2005) 13-28.

[21] N. Kuroki, Fuzzy bi-ideals in semigroups, Comment. Math. Univ. St. Pauli. 28(1979) $17-21$.

[22] N. Kuroki, Fuzzy semiprime quasi-ideals in semigroups, Inform. Sci., 75(1993) 201211.

[23] N. Kuroki, Fuzzy interior ideals in semigroups, J. fuzzy Math., 3(1995) 435-447.

[24] J. N. Mordeson, D. S. Malik and N. Kuroki, Fuzzy Semigroups, Springer Berlin, 2003.

[25] A. Lafi, DFIG control: A fuzzy approach, International Journal of Advanced and Applied Sciences, 6(019 107-116.

[26] Q. Mushtaq and S. M. Yusuf, On LA-semigroups, Alig. Bull. Math., 8(1978) 65-70.

[27] P. V. Protic and N. Stevanovic, AG-test and some general properties of AbelGrassmann's groupoids, Pure Math. appl., 6(1995) 371-383.

[28] S. A. Razak, D. Mohamad, I. I. Abdullah, A Group decision making problem using hierarchical based fuzzy soft matrix approach, International Journal of Advanced and Applied Sciences, 4((2017) 26-32.

[29] A. Rosenfeld, Fuzzy groups, J. Math. Anal. Appl., 35(1971) 512-517.

[30] T. Shah, N. Kausar, I. Rehman, Intuitionistic fuzzy normal subrings over a nonassociative ring, An. St. Univ. Ovidius Constanta, Vol. 20 (2012) 369-386. 
[31] T. Shah, N. Kausar, Characterizations of non-associative ordered semigroups by their fuzzy bi-ideals, Theoretical Computer Science, Vol. 529 (2014) 96-110.

[32] O. Ozer, S. Omran, On the generalized $\mathrm{C}^{*}$ - valued metric spaces related with Banach fixed point theory, International Journal of Advanced and Applied Sciences, 4(2017) $35-37$.

[33] L. A. Zadeh, Fuzzy sets, Inform. Control, 8(1965) 338-353. 